\title{
Evaluation of A Proprioceptive Program in A Patient with Cerebellar Ataxia After A Traumatic Brain Injury: Case Report
}

\author{
Giusppe Barone $^{1 *}$, Maria Grazia Benedetti ${ }^{2}$ and Laura Bragonzoni ${ }^{1}$ \\ ${ }^{1}$ Department for Life Quality Studies, University of Bologna, Bologna, Italy \\ ${ }^{2}$ Functional Recovery and Rehabilitation-IRCCS Istituto Ortopedico Rizzoli, Bologna, Italy
}

*Corresponding author: Maria Grazia Benedetti, Physical Therapy and Rehabilitation Unit, Istituto Ortopedico, Bologna, Italy.

To Cite This Article: Giusppe Barone, Maria Grazia Benedetti and Laura Bragonzoni. Evaluation of A Proprioceptive Program in A Patient with Cerebellar Ataxia After A Traumatic Brain Injury: Case Report. Am J Biomed Sci \& Res. 2021 - 12(1). AJBSR.MS.ID.001708. DOI: 10.34297/ AJBSR.2021.12.001708.

Received: 眥 January 18, 2021; Published: 眥 February 16, 2021

\begin{abstract}
Purpose: This study aims to evaluate the effect of innovative proprioceptive training in a patient with cerebellar ataxia due to Traumatic Brain Injury (TBI).

Materials and methods: A patient 20 years old, male with severe cerebellar ataxia, because of TBI was assessed and trained for proprioception impairment. The proprioception was evaluated during the stabilometric bipodalic test and the static monopodalic test by a mean of the Delos System (Delos, Turin, Italy). Parameters considered for these tests were: The Postural Instability, the Autonomy, and the Stability Index, which is a score (0$100 \%$ ) based on autonomy and postural instability.

Results: Postural Instability improved during the double stance test with open and closed eyes $\left(-0.32^{\circ} ;-0.8^{\circ}\right)$; Autonomy increased during the double stance test with open and closed eyes $(+22.3 \% ;+58 \%)$. The Stability Index measured during the single stance test also improved from $31.1 \%$ to $48.5 \%$ with Open Eyes and from $13.5 \%$ to $48.1 \%$ with closed Eyes.

Conclusion: The assessment of postural disorders in patients with cerebellar ataxia due to TBI is of clinical relevance. Results demonstrated that it was possible to improve the integration of the vestibular, visual, and proprioceptive systems in the maintenance of bipodalic stance.

Keywords: Traumatic Brain Injury, Cerebellar Ataxia, Proprioception, Balance, Case Report, Bipodalic Stance, Vestibular, Autonomy, Postural Instability, Dizziness, Cognitive Deficits, And Ataxia.
\end{abstract}

Abbreviations: Traumatic Brain Injury=TBI; Open Eyes=OE; Closed Eyes=CE; Stability Index=SI. Proprioceptive training program in ataxia

\section{Introduction}

Traumatic Brain Injury (TBI) is among the most common causes of neurologic disabilities in young adults [1,2]. The cerebellum is sometimes involved when the initial mechanical hit is direct to the cerebral cortex, with the following consequences: postural instability, dizziness, cognitive deficits, and ataxia [3]. Cerebellar ataxia, due to TBI, causes balance and coordination impairment that prevent proper walking and other movements with consequent disability and risk of falls [2]. Proprioception is a crucial factor to be considered in the treatment of these patients. Because of insufficient use of proprioceptive information, the deficit of proprioceptive sensory prevent relearning of essential function such as postural control and protective reflexes. Therefore, restoring neural connection is of great importance for the recovery of motor control. So, evaluation and treatment of proprioception in cerebellar ataxia is critical and should not be ignored [4].

Even though proprioceptive rehabilitation is usually strongly recommended in ataxic patients [1-3], there are no studies about that demonstrate its efficacy. Moreover, the techniques proposed for proprioception assessment are subjective, mainly based on clinical evaluation [5] while objective measurements of balance, like computerized dynamic posturography platform able to quantify the patient's functional deficits, should be recommended 
[6-9] as a reference method. In literature, the number of studies related to cerebellar ataxia is scant, due also at the wide range of postural disorders that may occur. It is also necessary an evidence-based guideline to define ataxia-specific expertise [10] in particular, focus on proprioceptive training. This case report aims to draw a new possibility of assessment and rehabilitation based on proprioception enhancement in a patient with cerebellar ataxia after traumatic brain injury using Delos Postural Proprioceptive System (Delos) based on the Riva Method [11].

\section{Material and Methods}

\section{Participant}

A 20-year-old male subject with previous TBI due to a traumatic incident occurred when he was 18 was examined. After the TBI the subject had a standard post-traumatic rehabilitation. Severe cerebellar Proprioceptive training program in ataxia, with gait impairment and unsteadiness remained. Walking was possible with slow speed and the help of a cane. Moreover, the subject has a monocular vision due to the previous retinoblastoma in the left eye. After baseline assessment, a 5-weeks training was performed. At the sixth week, the same baseline evaluations were carried out.

\section{Instrument}

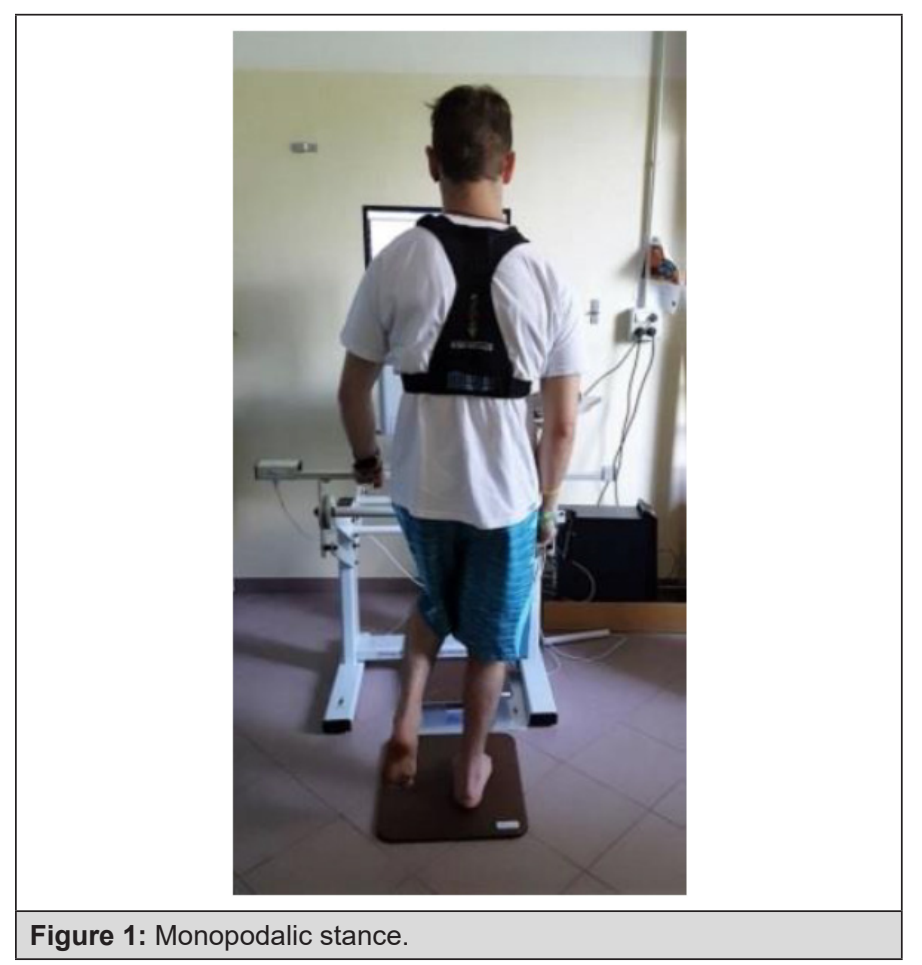

The stability tests and the rehabilitation training were performed using electronic postural proprioceptive stations (DPPS; Delos, Turin, Italy). It is an electronic station composed by a rocking or a static board, the Delos Vertical Controller (DVC-applied on a patient's sternum, records the movement on frontal and sagittal plans), the Delos Assistant Desk (DAD-a bar which provides support and records the contact times of the hands) and the display (shows visual feedback in real-time), connected to a personal computer with specific software (DPPS 6.0) for data elaborations. In this study, only the static board was used both for the double stance test and the single stance test monitoring the displacement of the projection of mass centre on the supporting plane, for a stability measure of the subject in static and dynamic conditions (Figure 1).

The stabilometric bipodalic test (double stance test) consists of 2 trials, one with Open Eyes (OE) and one with Closed Eyes (CE) while standing on a flat wooden surface with arms resting at sides. The subject is asked to stay still, trying to minimize sway. Each trial lasts 20 seconds, followed by a pause of 15 seconds. Parameters considered for this test were: The Postural Instability, measured in degrees, both in the $\mathrm{OE}$ and CE trials, and the Autonomy, corresponding to the time as a percentage spent without hand support on the bar. The static monopodalic test (single stance test) includes two trials with $\mathrm{OE}$ and 4 with $\mathrm{CE}$, and it is performed in single-leg stance (on the right and left limb). Parameters considered for this test were: The Stability Index and the Autonomy, both as a percentage. The Stability Index (SI) is a score (0-100\%) based on two components: Autonomy and average postural instability along the axes $\mathrm{X}$ and $\mathrm{Y}$.

\section{Exercise program}

A graduate trainer in sport science carried out ten training session of proprioceptive reconditioning performed two days a week. Every session had a duration of 45'-60' minutes and was articulated into five series of tasks, performed twice in a row, on the static board in double and single stance. The tasks consisted of following, in real-time on the monitor, moving targets with the eyes, trying to maintain balance. The visual target recording of these movements offers the patients under examination visualproprioceptive feedback. Proprioceptive training program in ataxia able to trigger, particularly at the sub-cortical level of the CNS, rapid reflexes of a corrective nature on posture control. The subject actively attended all the training sessions and adhered to the timetable.

\section{Results and Discussion}

Postural Instability measured during the double stance test increased after the training in the average from $0.99^{\circ}$ to $0.67^{\circ}$ with Open Eyes and from $1.41^{\circ}$ to $0.61^{\circ}$ with closed Eyes. Autonomy improved from $42.5 \%$ to $64.8 \%$ with Open Eyes and from $17.1 \%$ to $75.1 \%$ with closed Eyes. The Stability Index measured during the single stance test also improved from $31.1 \%$ to $48.5 \%$ with Open Eyes and from $13.5 \%$ to $48.1 \%$ with closed Eyes with increased Autonomy (Table 1). The assessment of postural disorders and the reprogramming of the proprioceptive system in patients with cerebellar ataxia due to TBI is of clinical relevance. Proprioceptive 
exercises have been used in the management of instability in cerebellar ataxia using common non-computerized Freeman-like platforms, and assessment methods are generally only qualitative [5]. The tool used in this study allows quantifying, with a score, the level of proprioception of the subject. The findings of this report indicate that significant improvements in proprioceptive and postural control can be obtained after specific training, personalized on the subject. Results demonstrated that it was possible to improve the integration of the vestibular, visual, and proprioceptive systems in the maintenance of bipodalic stance. The proprioceptive component solicited in the trials with closed eyes demonstrated to have a good margin of improvement. The same conclusion can be drawn for the monopodalic stance test in which the proprioceptive input, in the absence of visual control in the trial with EC, is integrated with the vestibular system both through a spinal reflex mechanism and cortical processing.

Table 1: Abbreviation: PI, postural instability, SI, stability index, AU, autonomy.

\begin{tabular}{|c|c|c|c|c|c|c|}
\hline \multirow{2}{*}{} & \multicolumn{3}{|c|}{ Open Eyes } & \multicolumn{3}{c|}{ Closed Eyes } \\
\cline { 2 - 7 } & PI & SI & AU & PI & SI & AU \\
\hline Pre-Reconditioning & 0.99 & 31.1 & 42.5 & 1.41 & 13.5 & 17.1 \\
\hline Post Reconditioning & 0.67 & 48.5 & 64.8 & 0.61 & 48.1 & 75.1 \\
\hline
\end{tabular}

\section{Conclusion}

The proposed rehabilitation training seems suitable and effective in improving postural control in cerebellar ataxia after TBI even if further follow up and a more extended training period can strongly support the outcome. However, further studies on a larger number of patients are required to refine the procedure, and to correlate the postural outcome with other indexes of functional performance and disability. Proprioceptive training program in ataxia. In the future, a specific proprioceptive protocol, with objective and quantitative measurements, could be included in a rehabilitation program for ataxic people. Moreover, will be significant recommend targeted exercises even after the rehabilitation phase, with clear and straightforward indications to be applied during daily life.

\section{Conflict of Interest}

The authors report no conflict of interest.

\section{References}

1. Sartor GC, Brickner L, (2014) A multidimensional physical therapy program for individuals with cerebellar ataxia secondary to traumatic brain injury: a case series. Physiother Theory Pract 30(2): 138-148.

2. Marsden J, Harris C, (2011) Cerebellar ataxia: pathophysiology and rehabilitation. Clin Rehabil 25(3): 195-216.

3. Müller SV, Amelie J, Bernd F, Reinhard D, Thomas F, et al. (2002) The effects of proprioceptive stimulation on cognitive processes in patients after traumatic brain injury. Arch Phys Med Rehabil 83(1): 115-121.

4. Demirci CS, Avcu F, Ayvat E, Kılınç M, Yıldırım SA, (2015) Proprioceptive Training in Neurological Diseases Proprioception. In Proprioception: The Forgotten Sixth Sense Edited by: Defne Kaya, OMICS Group eBooks Gull Ave USA.

5. Ayvat E, İyigün G, Kilinç M,Yildirim SA, (2015) Evaluation of Proprioception in Neurological Diseases In Proprioception: The Forgotten Sixth Sense Edited by: Defne Kaya, OMICS Group eBooks Gull Ave USA.

6. Wober C, W Oder, H Kollegger, L Prayer, C Baumgartner, et al. (1993) Posturographic measurement of body sway in survivors of severe closed head injury. Arch Phys Med Rehabil 74(11): 1151-1156.

7. Marquer A, Barbieri G, Pérennou D, (2014) The assessment and treatment of postural disorders in cerebellar ataxia: a systematic review. Ann Phys Rehabil Med 57(2): 67-78.

8. Kaufman KR, Robert HB, Li Shan C, Ann Rabatin, Allen W, et al. (2006) Comparison of subjective and objective measurements of balance disorders following traumatic brain injury. Med Eng Phys 28(3): 234239.

9. Petrò B, Papachatzopoulou A, Kiss RM (2017) Devices and tasks involved in the objective assessment of standing dynamic balancing-A systematic literature review. PLoS One 12(9): e0185188.

10. Synofzik M, Ilg W, (2014) Motor training in degenerative spinocerebellar disease: ataxia-specific improvements by intensive physiotherapy and exergames. Biomed Res Int 2014: 583507.

11. Riva D, Carlo Mamo, Mara Fanì, Patrizia S, Flavio R, et al. (2013) Single stance stability and proprioceptive control in older adults living at home: gender and age differences. J Aging Res 2013: 561695. 